Nervenarzt 2016 $87: 1271-1275$

DOI 10.1007/s00115-016-0232-8

Online publiziert: 26. Oktober 2016

(c) Der/die Autor(en) 2016. Dieser Artikel ist eine Open-Access-Publikation.

CrossMark

\author{
M. Uhr' $\cdot$ H. Tumani ${ }^{2,3} \cdot P$. Lange ${ }^{4}$ \\ ${ }^{1}$ Max-Planck-Institut für Psychiatrie, München, Deutschland \\ ${ }^{2}$ Fachklinik für Neurologie Dietenbronn, Schwendi, Deutschland \\ ${ }^{3}$ Neurologische Uniklinik im RKU, Ulm, Deutschland \\ ${ }^{4}$ Klinik für Neurologie UMG, Göttingen, Deutschland
}

\title{
Strategien der Liquorbefundung - integrierter Befundbericht
}

die Messungen der Werte aus gleichzeitig entnommenem Liquor und Serum mit anschließender Quotientenbildung notwendig ist. Der Vergleich dieser Liquor/ Serum-Quotienten untereinander und in speziell für die Liquordiagnostik validierten Auswertemodellen bringt die klinisch relevanten Aussagen [3, 4].

\section{Quotientenreihenfolge und -diagramm}

$\mathrm{Zu}$ den Hauptfragen der Liquoranalytik gehören die Fragen nach einer intrathekalen Immunglobulinproduktion, die definitionsgemäß einen entzündlichen Prozess im Zentralnervensystem (ZNS) nachweist. Da aber durch die immer vorhandene Diffusion der Serumproteine in den Liquor immer auch Immunglobuline in den Liquor gelangen, erfolgt die Beantwortung dieser Frage mit speziell entwickelten qualitativen und quantitativen Verfahren.

Bei den quantitativen Verfahren errechnet man, ob sich mehr Immunglobuline im Liquor befinden, als theoretisch durch Diffusion zu erwarten wäre, und geht in diesem Fall davon aus, dass sie dann im ZNS gebildet wurden. Dies erfolgt in Bezug auf den vom selben Patienten stammenden Albuminquotienten. Albumin wird nur in der Leber synthetisiert und dient damit als ideales Vergleichsprotein für die Immunglobuline.

Die einfachste aber unempfindlichste Auswertemethode vergleicht die Liquor/Serum-Quotienten (Q) von Albumin, Immunglobulin(Ig)G, IgA und IgM. Bedingt durch die molekülgrößenabhängige Diffusion muss das vom Molekül- radius kleinste Protein immer den größten Quotienten haben, also $\mathbf{Q}_{\text {Albumin }}>$ $\mathbf{Q}_{\mathrm{IgG}}>\mathbf{Q}_{\mathrm{IgA}}>\mathbf{Q}_{\mathrm{IgM}}$. Ist diese Reihenfolge unterbrochen, ist dies ein Hinweis auf eine entsprechende lokale Immunglobulinsynthese in ZNS.

Beispielsweise würde $\mathbf{Q}_{\operatorname{IgM}}>\mathbf{Q}_{\operatorname{IgA}}$ auf eine lokale IgM-Produktion hinweisen, auch wenn im Quotientdiagramm nach Reiber der IgM-Quotient im Referenzbereich liegen würde. Die gilt auch, abzüglich der Messschwankungen für Liquorproben, die mit artefiziellem Blut kontaminiert sind. Bei der artefiziellen Blutbeimengung werden alle Quotienten um den gleichen Betrag angehoben, die Reihenfolge ändert sich nicht.

Die sensitivere quantitative Methode ist die Auswertung Quotientendiagramm nach Reiber (• Abb. 1). Dabei kann über den individuellen Albuminquotienten des Patienten eine mögliche intrathekal gebildete Immunglobulinfraktion detektiert werden. Grenzwert $\left(\mathbf{Q}_{\text {lim }}\right)$ ist hier der höchste noch normale IgG-, IgA-, IgM-Quotient.

Die Immunglobulinquotienten werden mit dem Albuminquotienten über empirisch ermittelte und theoretisch bestätigte Formeln in Beziehung gesetzt (s. hierzu www.dgln.de und www. horeiber.de).

Mit Kenntnis des Albuminquotienten ist es möglich, vorherzusagen, in welchem Konzentrationsbereich 99\% der Immunglobuline liegen, wenn diese ausschließlich durch Diffusion in den Liquorraum gelangen. Bei höheren Quotientenwerten ist von einer zusätzlichen intrathekalen Synthese auszugehen. Besondere Vorsicht ist geboten, wenn die Anteil aus dem Blut stammen können, 


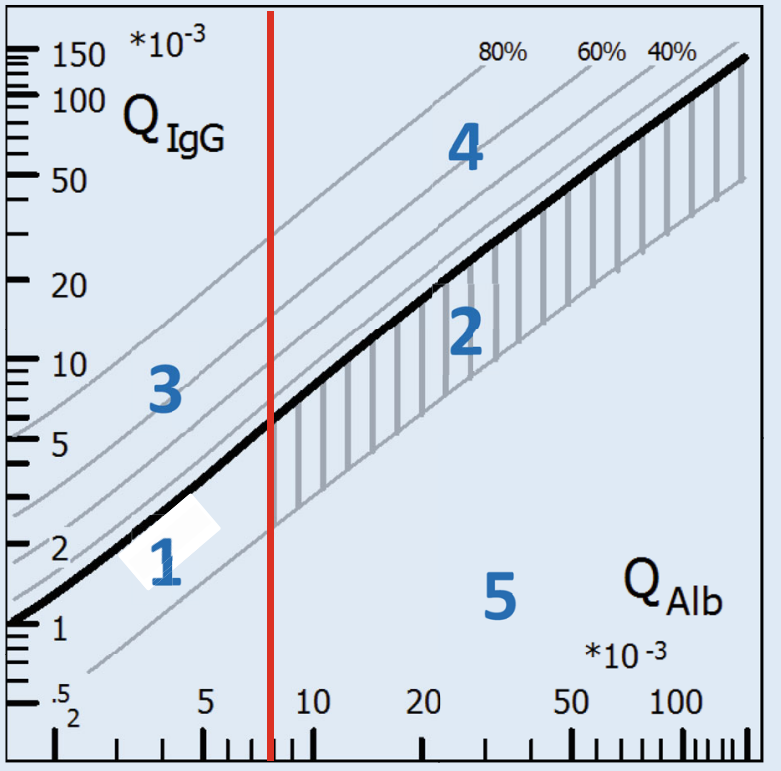

Abb. 1 A Quotientendiagramm für Immunglobulin(lg)G: In logarithmischer Auftragung sind der Albuminquotient gegen den IgG-Quotienten aufgetragen. Die dicke diagonal verlaufende Linie stellt den Q Lim $_{\text {(Grenz- }}$ wert) dar. IgG-Quotienten die oberhalb dieser Linie liegen haben eine intrathekale IgG-Synthese. Die vertikale rote Linie stellt den altersbezogenen Grenzwert für die Schrankenfunktion dar (Alter/15 + 4). Somit ergeben sich unterschiedliche Bereiche mit unterschiedlicher Befundinterpretation (und Erkrankungsbeispielen). 1 Normalbefund; 2 Schrankenfunktionsstörung (Guillain-Barré-Syndrom, Liquorflussbehinderung durch spinales Hindernis); 3 intrathekale IgG-Synthese (Multiple Sklerose); 4 Schrankenfunktionsstörung und intrathekale IgG-Synthese (Neuroborreliose); \% unplausibler Befund (High-dose-hook-Effekt, zu schnelle Punktion nach Immunglobulininfusion)

Konzentration unterhalb des erwarteten Bereichs liegt (•Abb. 1). Quotienten in diesem unplausiblen Bereich müssen unbedingt auf Mess- oder präanalytische Fehler hin überprüft werden.

\section{\) Das Quotientendiagramm ist unabhängig von den Messmethoden}

Zur Berechnung der Obergrenze $\mathbf{Q}_{\text {lim }}$ und der Untergrenze $\mathbf{Q}_{\text {low }}$ wird eine Hyperbelfunktion genutzt $\left(Q_{\operatorname{Igx}}=\left(\frac{\mathrm{a}}{\mathrm{b}}\right) \sqrt{Q_{\mathrm{alb}}^{2}+b^{2}}-\mathrm{c}\right)$. Die Konstanten $\mathrm{a}, \mathrm{b}$ und $\mathrm{c}$ sind für die jeweiligen Ober- und Untergrenzen für die jeweiligen Immunglobuline empirisch ermittelt worden. - Abb. 1 stellt das Quotientendiagramm für IgG mit den unterschiedlich zu interpretierenden Bereichen dar. Das Quotientendiagramm ist unabhängig von den Messmethoden. Das wird
Hinweise auf das vorliegende Krankheitsbild.

\section{Oligoklonale Banden}

Qualitative Verfahren zum Nachweis intrathekal gebildeter Immunglobuline nutzen die physikalischen Eigenschaften von Immunglobulinen. Das standardmäBig angewandte Verfahren zum Nachweis intrathekal gebildeten IgG sind die sog. oligoklonalen Banden (OKBs), die in der isoelektrischen Fokussierung (IEF) nachgewiesen werden (physikalische Eigenschaft ist hier die Wanderung im elektrischen Feld in Abhängigkeit vom pK-Wert des Immunglobulins). OKBs im Liquor, die nicht im Serum vorhanden sind, sprechen für eine intrathekale Synthese und damit für einen entzündlichen Prozess. Die IEF ist zum Nachweis einer ZNS-Entzündung empfindlicher als das Quotientendiagramm oder auch die Quotientenreihenfolge. Eine angezeigte IgG-Synthese im Quotientendiagramm und negative $\mathrm{OKBs}$ sind zunächst unplausibel. Dieses Ergebnis muss durch weitere Untersuchungen geklärt werden.

Bei einer artefiziellen Blutbeimengung nimmt die Sensitivität der OKBs ab. Isolierte OKBs im Liquor sind aber trotz einer artefiziellen Blutbeimengung als positiv zu werten. Auch hier wird ersichtlich, wie sehr sich die Ergebnisse untereinander bedingen.

Die Befundung der IEF erfolgt entsprechend der internationalen Vorgaben (Typ I bis Typ V nach Andersson et al. [1]; s. auch www.dgln.de [2]). Die Ergebnisse der OKBs sind im integrierten Gesamtbefund darzustellen und $\mathrm{zu}$ interpretieren.

\section{Antikörperindizes} und IgM, sortiert nach der Radiusgröße der Immunglobuline untereinander, ist als Standard etabliert. Dadurch ergibt sich auf einen Blick die Möglichkeit der Erkennung krankheitstypischer Befundmuster. Diese Mustererkennung der acht Messgrößen (Liquor- und Serumwerte von Albumin, IgG, IgA und IgM) in den spezialisierten Diagrammen ermöglicht es dem Labor, die Messwerte rasch auf Plausibilität zu überprüfen. Zudem liefert diese Mustererkennung wesentliche
Die Ergebnisse des Nachweises einer intrathekalen Synthese spezifischer Antikörper (Antikörperindizes $=$ AI-Werte) gegen ein zu untersuchendes Antigen (Erreger) gehört in den integrierten Befundbericht.

Man geht bei dieser Untersuchung davon aus, dass spezifische Antikörper einer bestimmten Immunglobulinklasse (IgG, IgA, IgM) sich bezüglich ihrer Eigenschaften und damit auch ihres Dif- 
fusionsverhaltens ähnlich verhalten wie alle übrigen Antikörper dieser Klasse zusammen. Somit wird der spezifische Liquor/Serum-Antikörper-Quotient mit dem Liquor/Serum-Quotienten des Gesamtimmunglobulins verglichen. Ist dieser spezifische Quotient größer als der des Gesamtimmunglobulins (abzüglich der Messgenauigkeit), geht man von einer spezifischen intrathekalen Antikörpersynthese im ZNS aus. Zu bedenken ist, dass immer mit den aus dem Blut kommenden Immunglobulinen verglichen werden muss. Deshalb werden bei der Berechnung der Antikörperindizes zwei Auswertesituationen unterschieden [5].

Fall 1. Liegt der Quotient des Gesamtglobulins im Reiber-Quotientendiagramm unterhalb der Differenzierungslinie zum Nachweis einer lokalen Synthese (unterhalb $\mathrm{QIgX}_{\mathrm{lim}}$ ), kann zur Berechnung des AI-Wertes der gemessene Liquor/ Serum-Quotient des Gesamtimmunglobulins genommen werden.
Berechnung Fall 1: AI $=Q_{\text {spezifisch }} /$ $\mathrm{Q}_{\text {gesamt }}$ wenn $\mathrm{Q}_{\text {gesamt }}<\mathrm{Q}_{\text {lim }}$

Fall 2. Liegt aber im Quotientendiagramm eine intrathekale Gesamtsynthese vor, wird der AI-Wert mit den QLim (den höchsten noch normalen IgX-Quotienten bezogen auf den individuellen Albuminquotienten) berechnet.

Berechnung Fall 2: $\mathrm{AI}=\mathrm{Q}_{\text {spezifisch }} / \mathrm{Q}_{\text {lim }}$ wenn $Q_{\text {gesamt }}>\mathrm{Q}_{\text {lim }}$

Bei der artefiziellen Blutbeimengung wird die Sache komplizierter. Man darf dann nicht auf den $\mathrm{Q}_{\text {lim }}$ beziehen, wenn der Gesamtimmunglobulinquotient größer $\mathrm{Q}_{\text {Lim }}$ ist, da die Immunglobuline möglicherweise nicht intrathekal gebildet wurden, sondern ein Teil mit dem artefiziellen Blut ungefiltert in den Liquor gelangt ist. Das passiert aber auch mit den spezifischen Antikörpern und würde somit durch einen Bezug auf den $\mathbf{Q}_{\text {lim }}$ (im Sinne einer „Korrektur") zu falsch-positiven Befunden führen. Werden mehrere Antikörper gegen spezifische Antigene gleichzeitig untersucht, ergibt sich hieraus die Möglichkeit durch besondere Befundmuster methodische Schwierigkeiten und Hinweise auf chronisch-entzündliche Prozesse im ZNS zu erkennen. Auch hier haben Betrachtungen von Einzelbefunden deutliche Limitationen gegenüber einer umfangreicheren multiparametrischen Diagnostik, da letztere auch komplexere Befundinterpretationen ermöglichen.

\section{Zellzahl und Zytologie}

Zum integrierten Befundbericht gehören eine verlässliche Zell- und zwingend auch Erythrozytenzahl. Auch bei normaler Zellzahl ist eine Zytologie durchzuführen, um Hinweise auf entzündliche Prozesse, Blutungen, Tumoren etc. $\mathrm{zu}$ finden. Das Zellbild liefert wichtige Hinweise für die Plausibilität der Befunde und der diagnostischen Interpretation (s. auch [8] in diesem Heft von Der Nervenarzt).

Hier steht eine Anzeige. 
Neben der Schrankenfunktionsstörung (erhöhter $\mathbf{Q}_{\mathrm{Alb}}$ ) ist die Zellzahl ein wichtiger Marker, der den Verlauf einer Erkrankung anzeigt. Werden Patienten mehrmalig punktiert, ergeben sich zum Teil Veränderungen in der Befundkonstellation, die dann erst durch die Gesamtbefundung ihren Wert zeigen. Auch ist anzuführen, dass der Zeitpunkt der Punktion (diagnostische Punktion, Verlaufspunktion) wichtige Informationen in Ergänzung zu klinischen Angaben und Fragestellung darstellen.

\section{Probleme und Lösungen bei artefizieller Blutbeimengung}

Bei einer intrazerebralen/spinalen Blutung geben Erythrozytenzahl, Hämoglobin und Ferritin, Erythrophagen und Hämosiderophagen im Liquor wichtige Hinweise. Eine Korrektur der Liquorproteinwerte ist nur bei einer artefiziellen Blutung sinnvoll und möglich. Dabei dient die Erythrozytenzahl als Indikator für die Menge an Blut, die in den Liquor eingetragen wurde. Bedenkt man die beträchtliche Differenz der Konzentrationen zwischen Liquor und Serum, so ist dies von größter Relevanz und bei der Befundung zwingend $\mathrm{zu}$ berücksichtigen. Beispielhaft sei hier das IgM angeführt. Die Serum-IgM-Konzentrationen sind ca. 3300-mal so hoch wie die im physiologischen Liquor. Dies bedeutet, dass sich die IgM-Liquorkonzentration um das Doppelte erhöht, wenn nur ein Dreitausendstel Serum artefiziell in den Liquor gelangt. Dies entspricht ca. 1000 Erythrozyten $/ \mu$ l. Solche oder höhere Blutbeimengungen werden bei Punktionen der Liquorräume in $10-20 \%$ der Fälle beobachtet.

Auch ist zu bedenken, dass Untersuchungen vielfach aus einer einzelnen Portion der Liquorgewinnung gemacht werden. Eine Aufteilung/Alliquotierung sollte erst durch das Labor aus der Gesamtmenge erfolgen. Die häufig angewandte Praxis, dass mehrere Röhrchen unmittelbar bei der Punktion gefüllt und in unterschiedliche Labore verschickt werden, ist fehlerträchtig und kann zu Fehlinterpretation führen. Die Gefahr unterschiedlicher Blutbeimengungen in den Liquorportionen und die sich daraus er- gebenen Fehlern bei dem anschließenden Verrechnen der Einzelwerte mit der Konsequenz einer Falschbefundung, ist erheblich.

Beispiel. Im ersten Röhrchen sind 1000 Erythrozyten/ $\mu$ und es wird der spezifische Borrelien-IgM-AntikörperLiquor/Serum-Quotient bestimmt; im zweiten Röhrchen besteht keine Blutbeimengung mehr und es wir der GesamtIgM-Quotient gebildet; bei der Berechnung ergibt sich dann für den BorrelienIgM-Antikörperindex mit 2 ein falschpositiver Index, weil im ersten Röhrchen das spezifische Borrelien-IgM durch den Bluteintrag verdoppelt wurde.

\section{Zusammenfassende Beurteilung}

Auch wenn in diesem Artikel die Liquoranalytik nicht ausführlich erklärt werden kann, sollen die angeführten Beispiele klarmachen, wie sehr die Einzelbefunde voneinander abhängen und sich gegenseitig bedingen können. Daher erlaubt die Verwendung des integrierten Gesamtbefundes dem erfahrenen Laborarzt auch die Einzelergebnisse auf ihre Richtigkeit zu überprüfen. Bestimmte Befundmuster sind ganz typisch für Messfehler (High-dose-hook-Effekt usw.) und präanalytische Fehler (Blutbeimengung, zu frühe Punktion nach Immunglobulininfusion, Plasmapherese usw.). Dem klinisch behandelnden Arzt kann die Erkennung von Befundmustern wesentliche differenzialdiagnostische Hinweise geben.

\section{I) Alle aus dem Liquor gemessenen Daten sind einer Gesamtbeurteilung zuzuführen}

Somit sollte zusammenfassend nochmals festgehalten werden, dass es sehr viel Evidenz dafür gibt, alle aus dem Liquor gemessenen Daten zusammenzufassen und einer Gesamtbeurteilung zuzuführen. Auch sollten dabei die Mittel genutzt werden, die eine Befundung erleichtern und übersichtlich gestalten lassen wie z. B. Quotientendiagramme. Auf der anderen Seite ist auf überflüssi-
Nervenarzt 2016 $87: 1271-1275$

DOI 10.1007/s00115-016-0232-8

(c) Der/die Autor(en) 2016. Dieser Artikel ist eine Open-Access-Publikation.

\section{Uhr $\cdot$ H. Tumani $\cdot$ P. Lange}

\section{Strategien der Liquorbefundung - integrierter Befundbericht}

\section{Zusammenfassung}

Die Liquoranalytik erfordert eine zusammenfassende Beurteilung aller Einzelbefunde in einem integrierten Gesamtbefund, damit eine zuverlässige und diagnosespezifische Befundaussage erzielt werden kann. Diese Befundungsstrategie erlaubt das Erkennen krankheitstypischer Befundmuster sowie auch Plausibilitätskontrollen zur Vermeidung von Analytikfehlern. Der Gesamtbefund besteht aus 1) einem Liquorgrundprogramm mit zytologischen und proteinchemischen Parametern, 2) einem erweiterten Programm mit Spezialparametern für Erregernachweise sowie Marker für Neurodegeneration und 3) einer abschließenden Interpretation mit methodischer und klinischer Beurteilung.

\section{Schlüsselwörter}

Zytologie - Liquorpoteine - Serumglobuline . Albumine $\cdot$ Immunglobuline

Strategies for cerebrospinal fluid analysis - Integrated results report

\section{Abstract}

Cerebrospinal fluid (CSF) analysis requires a combined assessment of all individual test findings in an integrated total report in order to achieve a reliable and specific diagnostic conclusion. Such a standard assessment strategy allows the identification of diseasetypical result patterns and plausibility checks to avoid analytical errors. The integrated total report consists of 1) a basic CSF program with cytological and protein chemical parameters, 2) an expanded CSF program with special parameters for detection of pathogens and markers of neurodegeneration and 3) a final contextual interpretation considering methodological and clinical aspects.

\section{Keywords}

Cytology · Cerebrospinal fluid proteins - Serum globulins - Albumins . Immunoglobulins 
ge verwirrende und nicht zielführende Informationen (unangemessene Referenzwerte) oder graphische Aufarbeitungen zu verzichten. Eine Interpretation sollte dem klinischen Kollegen die wesentlichen Hauptaussagen eindeutig mitteilen. Dies könnten z. B. bakterielle Infektion, chronische Entzündung, intrathekale Antikörperproduktion, ältere Blutung, pathologische Demenz/ Destruktionsmarker usw. sein.

Leider ist die Realität eine andere und in nicht wenigen Kliniken/Praxen müssen die tätigen Kollegen mit Einzelbefunden unabhängig vom entsprechenden Kontext zurechtkommen. Dabei bleiben nicht selten Informationen auf der Strecke oder können nicht richtig vom behandelnden Kollegen zum Wohle des Patienten eingesetzt werden. Auch wenn der finanzielle Druck im Gesundheitswesen sehr groß ist, sollten die oben benannten Gegebenheiten beachtet werden und die Einzelparameter nicht nur nach Kostengründen verschickt werden. Dazu kommt, dass eine integrierte Befundungsleistung nicht honoriert wird und damit vielerorts diese Zusatzleistungen nicht darstellbar sind. Auch ergibt sich häufig die Situation, dass mehrere Laboratorien an den Liquormessungen beteiligt werden und jeweils ihre eigenen Befunde einzeln zurückschicken. Die Empfehlung der DGLN (Deutsche Gesellschaft für Liquoranalytik und klinische Neurochemie e. V.) beinhaltet in solch einem Fall, die Einzelbefunde in einem integrierten Liquorbefund in der anfordernden Abteilung zusammenzutragen. Um möglichst viele in die Lage $\mathrm{zu}$ versetzen, diese Tätigkeiten auch in den punktierenden Fächern durchführen zu können, werden von der DGLN, Liquor- und Befundungskurse angeboten. Darüber hinaus gibt es die Möglichkeit, seine Kenntnisse nach Durchlauf eines speziellen Kurrikulums und Prüfung durch die Erlangung eines klinischen Zertifikates der DGLN zu belegen. Weitere Informationen können der Website der DGLN (www.dgln.de) entnommen werden, auch der Vorsitzende der Weiterbildungskommission gibt hierzu Auskunft.

\section{Fazit für die Praxis}

In der Liquordiagnostik ist eine zusammenfassende Beurteilung aller Einzelbefunde in einem integrierten Gesamtbefund sehr sinnvoll, um eine diagnosespezifische Befundaussage zu ermöglichen. Der Gesamtbefund besteht aus:

- dem Liquorgrundprogramm (Zellzahl, Erythrozytenzahl, Zytologie, Gesamteiweiß, Laktat im Liquor, Glukosequotient (wenn Glukose dann nur Serum- und Liquorwerte im Kontext), ggf. Ferritin, Liquor/SerumQuotienten von Albumin, IgG, IgA und $\lg M$, bevorzugt mit graphischer Darstellung im Quotientendiagramm und oligoklonalen Banden),

- dem erweiterten Programm (direkte oder indirekte Nachweise von Erregern mittels PCR oder Antikörperindizes, Erregernachweis [Bakteriologie], freie Leichtketten, CXCL13, Demenz/Destruktionsmarker [tTau, pTau, $\beta$-Amyloid 1-42 etc., GFAP, Neurofilamente, Orexin und weitere ZNS-spezifische Markerproteine] und - einer abschließenden deskriptiven Befundung (Hinweis auf Entzündung, Blutung, Schrankendysfunktion, Meningeosis, degenerativer Prozess) und Interpretation mit methodischer und klinischer Beurteilung.

\section{Korrespondenzadresse}

\section{PD Dr. Dr. M. Uhr}

Max-Planck-Institut für Psychiatrie Kraepelinstr. 2-10, 80804 München, Deutschland

uhr@psych.mpg.de

Open access funding provided by Max Planck Society.

\section{Einhaltung ethischer Richtlinien}

Interessenkonflikt. M. Uhr, H. Tumani und P. Lange geben an, dass kein Interessenkonflikt besteht.

Dieser Beitrag beinhaltet keine von den Autoren durchgeführten Studien an Menschen oder Tieren.

Open Access Dieser Artikel wird unter der Creative Commons Namensnennung 4.0 International Lizenz (http://creativecommons.org/licenses/by/4.0/deed. de) veröffentlicht, welche die Nutzung, Vervielfältigung, Bearbeitung, Verbreitung und Wiedergabe in jeglichem Medium und Format erlaubt, sofern Sie den/die ursprünglichen Autor(en) und die Quelle ordnungsgemäßnennen, einen Linkzur Creative Commons Lizenz beifügen und angeben, ob Änderungen vorgenommen wurden.

\section{Literatur}

1. Andersson M, Alvarez-Cermeño J, Bernardi G, Cogato I, Fredman P, Frederiksen J, Fredrikson S, Gallo P, Grimaldi LM, Grønning M et al (1994) Cerebrospinal fluid in the diagnosis of multiple sclerosis: A consensus report. J Neurol Neurosurg Psychiatr 57(8):897-902

2. Petereit HF, Sindern E, Wick M (Hrsg) (2007) Leitlinien und Methodenkatalog der DGLN. Springer, Heidelberg, Berlin, New York (siehe auch www.dgln.de)

3. Reiber H (1994) Flow rate of cerebrospinal fluid (CSF) - a concept common to normal blood-CSF barrier function and to dysfunction in neurological diseases. J Neurol Sci 122:189-203

4. Reiber H, Felgenhauer K (1987) Protein transfer at the blood cerebrospinal fluid barrier and the quantitation of the humoral immune response within the central nervous system. Clin Chim Acta 163:319-328

5. Reiber H, Lange P (1991) Quantification of virusspecific antibodies in cerebrospinal fluid and serum: sensitive and specific detection of antibody synthesis in brain. Clin Chem 37(7):1153-1160

6. Ruprecht K, Tumani H (2016) Liquordiagnostik bei Multipler Sklerose. Nervenarzt. doi:10.1007/s00115-016-0220-z

7. Spreer A, Rauer S, Wilking H, Fingerle V (2016) Herausforderung Neuroborreliose. Nervenarzt. doi:10-1007/s00115-016-0224-8

8. Wick M, Gross CC, Isenmann S, Strik H (2016) Liquorzytologie. Nervenarzt. doi:10.1007/s00115016-0219-5 